\title{
Medical Imaging Informatics
}

\author{
A.A.T. Bui and R.K. Taira, eds.
}

New York, NY: Springer, 2010, 446 pages, $\$ 169$

The Society for Imaging Informatics in Medicine defines imaging informatics as follows:

\begin{abstract}
"Imaging informatics touches every aspect of the imaging chain from image creation and acquisition, to image distribution and management, to image storage and retrieval, to image processing, analysis and understanding, to image visualization and data navigation; to image interpretation, reporting, and communications. The field serves as the integrative catalyst for these processes and forms a bridge with imaging and other medical disciplines." (1)
\end{abstract}

Medical Imaging Informatics takes this exact approach by introducing the reader to the multifaceted discipline of imaging informatics and following the process of patient care along the way. The text is divided into 4 parts, and each part is subdivided into 2 or 3 chapters. The text proceeds in a logical fashion, with chapter 1 providing an introduction to medical imaging informatics. The remainder of part I addresses the imaging examination, including imaging modalities and the content of medical images, specifically anatomy and physiology. Although chapter 2 is titled "A Primer on Imaging Anatomy and Physiology," almost half of the chapter is devoted to a review of imaging modalities, including a detailed focus on the physics of these modalities. Part II also includes a discussion of the integration of imaging into the patient record, covering data standards, electronic medical records, information architectures, and data visualization. Included in part II are excellent discussions of the different types of electronic medical records, Digital Imaging and Communications in Medicine (DICOM), Health Level 7, and common network topologies. Part III addresses problems associated with extracting information from images and organizing this information so it can be searched and mined and the maximum amount of data extracted. Chapter 6, an educational exposition of natural language processing of medical reports, is well written and moves the reader through the chapter fluidly. Part IV, the final part of the book, covers medical decision making, including disease models, study design, and study evaluation. A lengthy discussion of Bayesian belief networks is featured prominently in part IV.

Although part of each chapter or subsection is used to introduce the topics of discussion, the interested reader would do well to at least be familiar with, and preferably have some formal education in, the areas of computer science and

COPYRIGHT @ 2010 by the Society of Nuclear Medicine, Inc. DOI: $10.2967 /$ jnumed. 110.078980 advanced mathematics to fully comprehend many of the topics discussed in the text. It is likely that a casual reader would quickly become lost in the complex mathematics and technical jargon that are scattered throughout the text, whereas an imaging informatics researcher would find the same information quite useful and understandable. The editors address this issue in the preface. However, this is not a text for an average hospital imaging informatics professional. The topics covered later in the text are outside the scope of what a PACS or radiology information system administrator would need to know and understand. Also, any discussion of practical imaging informatics, such as the role of quality control and quality improvement in imaging informatics and vice versa, is absent. This text is geared toward researchers in the field and toward radiologists with a serious interest in the future of informatics.

Strengths of the text include the frequent real-world examples of the concepts discussed, which are directly related to current research and developments in imaging informatics. My favorite example concerned the TraumaSCAN system, which uses Bayesian belief networks to predict symptoms and conditions arising from gunshot wounds, which are identified by a physician on a human model.

Also of note are the extensive reference lists at the end of each chapter, making the text function not only as an educational resource but also as a literature review. These reference lists will be invaluable to researchers in the field. It is evident that the authors and editors have worked hard to provide both breadth and depth of information in this text. A substantial amount of discussion is devoted to current informatics challenges, including efforts to standardize image data acquisition and processing to facilitate comparisons across institutions, for example as part of multicenter trials.

Another topic I particularly enjoyed was the discussion of alternatives to existing medical record formats and medical data storage methods. Among the alternatives introduced were the "problem-oriented medical record," whereby a patient's medical information is organized into a series of ongoing and resolved problems; the "personal medical record," which incorporates additional learning opportunities and resources to help patients understand their medical history and condition; and the concept of the "phenomenoncentric data model," wherein medical problems are treated similarly to scientific experiments, with the problem being 
the phenomenon a physician wishes to explain by following the scientific method.

This text would benefit from a more comprehensive editorial review and a review of the medical physics topics for accuracy by a medical physicist. The readability of the text is hampered by an overabundance of parenthetical statements, and several conspicuous misspellings and misused words appear throughout the text. Many figure captions refer to colors, but all of the figures are in black and white; also, several figures are not of sufficient resolution or size. There are other significant errors that may confuse the reader: for example, the roles of the service class provider and service class user in the DICOM storage service class are reversed in chapter 3 . In addition, the sections of chapter 2 that address imaging modalities and imaging physics include numerous errors and warrant careful review by a medical physicist.

Finally, at times the text seems to stray from its intended purpose and includes seemingly irrelevant or extraneous information. For example, an entire paragraph in chapter 2 was devoted to explaining the physiology of "having the wind knocked out of you" and 2 full pages in chapter 5 are devoted to a detailed discussion of dual-energy subtraction radiography, which seems out of proportion to its importance to the purpose of the text.

On finishing this text, the reader is left almost overwhelmed at the scope of the problem facing informatics today: how do we standardize, integrate, and mine medical information; make decisions based on this information; and evaluate our decisions? The editors and authors have done an excellent job of offering answers to these questions and suggesting how the solutions to each piece of the puzzle can be assembled to benefit physicians and patients.

\section{REFERENCE}

1. Society for Imaging Informatics in Medicine Web site. "Imaging Informatics" page. Available at: http://www.siimweb.org/index.cfm?id=324. Accessed July 16, 2010.

\section{A. Kyle Jones, PhD}

U.T. M.D. Anderson Cancer Center

1515 Holcombe Blvd., Unit 1352

Houston, TX 77030-4009

E-mail: kyle.jones@mdanderson.org 